\title{
The Influence of Changes in Loading Patterns on Left Ventricular Relaxation in Humans
}

\author{
Toshiro Iketani, MD; Kenji Takazawa, MD; Chiharu Ibukiyama, MD
}

\begin{abstract}
This clinical investigation was designed to determine the effect of changes in loading patterns on left ventricular (LV) relaxation when heart rate was maintained constant. Not only were changes noted in total load or time in which load is changed, but also the contour of the ascending aortic systolic pressure wave. Twenty patients were studied. LV and ascending aortic pressure were measured by a multisensor catheter under baseline conditions (C) and after an intravenous injection of $2.5 \mu \mathrm{g}$ angiotensin (A) and sublingual administration of $0.3 \mathrm{mg}$ nitroglycerin $(\mathrm{N})$. A bipolar pacing catheter was placed in the right atrium to maintain a constant heart rate throughout the protocol. The augmentation index (AI), which characterizes the contour of the ascending aortic systolic pressure wave, was defined as the ratio of the height of the late systolic shoulder/peak to that of the early systolic shoulder/peak in the pulse. The rate of isovolumic LV pressure decline was calculated as a time constant (Tau). Ascending aortic systolic pressures $(\mathrm{mmHg})$ were $127 \pm 29(\mathrm{C}), 158 \pm 20(\mathrm{~A})$ and $109 \pm 15(\mathrm{~N})$. AI were $1.61 \pm 1.14$ (C), 2.08 $\pm 1.11(\mathrm{~A})$ and $1.27 \pm 1.14(\mathrm{~N})$. Tau values $(\mathrm{msec})$ were $49 \pm 4(\mathrm{C}), 54 \pm 4(\mathrm{~A})$ and $45 \pm 5(\mathrm{~N})$. Tau was prolonged proportionally with increasing AI $(\mathrm{p}<0.001, \mathrm{r}=0.64)$. It was concluded that late systolic pressure augmentation in the ascending aorta is one important factor that influences the rate of isovolumic left ventricular pressure decline in humans. (Jpn Circ J 1998; 62: 581-585)
\end{abstract}

Key Words: Afterload; Augmentation index; Blood pressure; Diastole; Left ventricular relaxation

$\mathbf{S}$ everal investigators have reported that increases in afterload or systolic pressure will prolong the rate of isovolumic left ventricular (LV) pressure decline (Tau)!-3 In contrast, the relaxation rate has also been reported to be accelerated when afterload or systolic pressure increase.,5 Zile et al noted the time during contraction that load is changed! Loads applied early in contraction (within the first two-thirds of the ejection time) generally prolong Tau, whereas loads applied late in contraction (during the late one-third of the ejection time) generally cause Tau to be shortened. Hori et al also demonstrated that Tau is variably affected by the timing of peak LV pressure and the timing of end ejection 3,7 Thus Tau is affected by the magnitude of the change in load and the time during contraction in which the load is changed. Moreover, several investigators reported that increased heart rate (HR) is a factor that shortens Tau, ${ }^{4,8-10}$ Starling et al demonstrated that Tau is unaffected by modest alterations in loading conditions in humans when HR is maintained constant? This present investigation was therefore designed to determine the effect of changes in loading patterns or blood pressure on LV relaxation when HR was maintained constant. We noted not only magnitude of the change in systolic blood pressure or time during contraction in which the load is changed, but also the contour of the systolic pressure wave. We assessed the loading pattern as the ascending aortic augmentation index (AI) $)^{11-13}$ which is defined as the ratio of the height of the late systolic shoul-

(Received February 9, 1998; revised manuscript received March 25, 1998; accepted March 28, 1998)

The Second Department of Internal Medicine, Tokyo Medical College, Tokyo, Japan

Mailing address: Kenji Takazawa, MD, The Second Department of Internal Medicine, Tokyo Medical College, 6-7-1 Nishishinjuku, Shinjuku-ku, Tokyo 160-0023, Japan der/peak to that of the early systolic shoulder/peak in the pulse ${ }^{14}$ The $\mathrm{AI}$ is an indicator of the vascular load, ${ }^{11,13,15}$ the vascular characteristic, ${ }^{11-13}$ and the left ventricular afterload ${ }^{2,15}$ Moreover the AI characterizes the contour of the ascending aortic systolic pressure wave.

\section{Methods}

\section{Patients}

Twenty patients ( 18 men and 2 women) who underwent diagnostic cardiac catheterization were studied. Their ages ranged from 24 to 76 years old with a mean age of $55 \pm 14$ (1 standard deviation). Ten patients had myocardial infarction, 4 had angina pectoris, 3 had myocarditis, and 3 had chest pain syndrome without organic cardiac abnormalities. All procedures were approved by the Ethics Committee of Tokyo Medical College Hospital. Informed consent was obtained from all patients.

\section{Study Protocol}

Left ventricular and ascending aortic pressure were measured by a micro tip catheter (SVPC684D, Millar Instruments, Houston, USA); both were recorded simultaneously on an FM tape recorder (XR-30, TEAC Co, Tokyo, Japan). Waves were analyzed by a signal processor (Sanei 7T18A, Nippon Electric Co, Tokyo, Japan). Cardiac output was measured using a Swan-Ganz catheter and the thermodilution method. In addition, a bipolar pacing catheter was placed in the right atrium to maintain a constant HR throughout the protocol. Measurements were carried out in the baseline condition and when the systolic pressure increased by about $25 \%$ after intravenous injection of $2.5 \mu \mathrm{g}$ angiotensin. After the systolic blood pressure returned to control levels, $0.3 \mathrm{mg}$ sublingual nitroglycerin was administered and measurements were made 5 min later (Fig 1). 

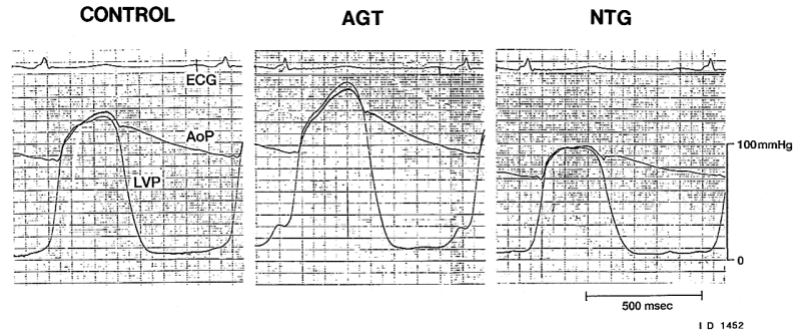

Fig 1. Examples of pressure recordings of the left ventricle and the ascending aorta in the patient's baseline conditions (CONTROL), after angiotensin infusion (AGT) and after sublingual administration of nitroglycerin (NTG). The ascending aortic and left ventricular pressure increased after AGT, especially in late systole. After NTG, the ascending aortic and left ventricular pressure decreased and late systolic components of both pressure waves decreased markedly. AoP, ascending aortic pressure; LVP, left ventricular pressure; ECG, electrocardiogram.

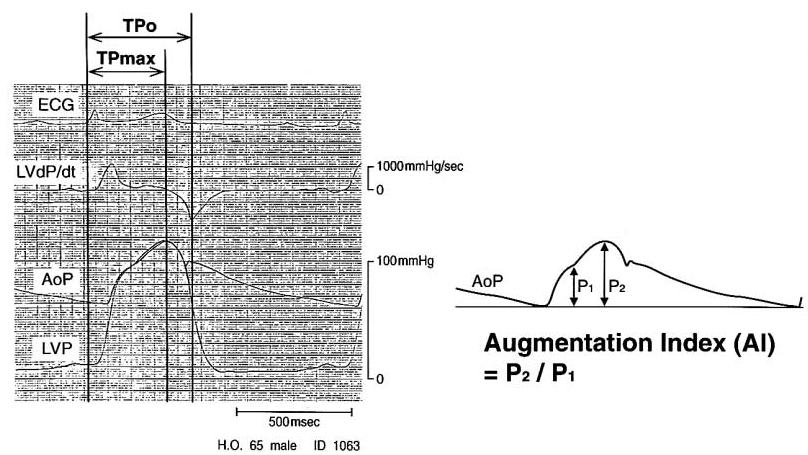

Fig 2. The augmentation index (AI) was defined as the ratio of the height of the late systolic shoulder/peak to that of the early systolic shoulder/peak in the pulse. $\mathrm{P}_{1}$, the height of the early systolic shoulder/peak in the pulse; $\mathrm{P}_{2}$, the height of the late systolic shoulder/peak in the pulse; AoP, ascending aortic pressure; LVP, left ventricular pressure; $\mathrm{LVdP} / \mathrm{dt}$, the first deviative of LVP; ECG, electrocardiogram; TPmax, the times from onset of the $\mathrm{R}$ wave on electrocardiogram to peak LV pressure; TPo, the times from onset of the $\mathrm{R}$ wave on electrocardiogram to minimal $\mathrm{dP} / \mathrm{dt}$.

\section{The Timing of the Onset and the Time Constant of Isovolumic LV Relaxation}

The times from the onset of the $\mathrm{R}$ wave on electrocardiogram to minimal $\mathrm{dP} / \mathrm{dt}$ (TPo), which is very close to the time of the onset of isovolumic relaxation or end of ejection ${ }^{16}$ were obtained from the pressure data. The time constant (Tau) of isovolumic LV pressure decay from minimal $\mathrm{dP} / \mathrm{dt}$ to $10 \mathrm{mmHg}$ above $\mathrm{LV}$ end-diastolic pressure of the following beat was calculated from the following equation,

$$
\mathrm{P}(\mathrm{t})=\mathrm{P} 0 \exp ^{-\mathrm{t} / \mathrm{Tau}}
$$

where $P(t)$ is the left ventricular pressure at any time $(t)$, $\mathrm{P} 0$ is the initial left ventricular pressure during isovolumic relaxation, and exp is the base of the natural logarithm! ${ }^{17}$ Tau values were obtained from the least-squares method.

\section{Indices of Loading Patterns}

Three indices that characterize the loading patterns were determined as follows.

The Time of End Ejection (TPo): The time from the onset of the $\mathrm{R}$ wave on electrocardiogram to minimal $\mathrm{dP} / \mathrm{dt}$ (TPo), which is assumed to be the time of end ejection, was
Table 1 Hemodynamics

\begin{tabular}{lllccc}
\hline \hline & $\begin{array}{c}S P \\
(\mathrm{mmHg})\end{array}$ & $\begin{array}{c}\text { DP } \\
(\mathrm{mmHg})\end{array}$ & $\begin{array}{c}E D P \\
(\mathrm{mmHg})\end{array}$ & $\begin{array}{c}H R \\
(\text { beats } / \mathrm{min})\end{array}$ & $\begin{array}{c}S V I \\
\left(\mathrm{ml} / \mathrm{m}^{2}\right)\end{array}$ \\
\hline Control & $127 \pm 29$ & $76 \pm 13$ & $15 \pm 3$ & $70 \pm 13$ & $41 \pm 5$ \\
AGT & $158 \pm 20^{*}$ & $93 \pm 15^{*}$ & $23 \pm 5 *$ & $70 \pm 13$ & $39 \pm 5$ \\
NTG & $109 \pm 15^{*}$ & $73 \pm 13^{*}$ & $10 \pm 4^{*}$ & $71 \pm 13$ & $39 \pm 6$ \\
\hline
\end{tabular}

The pressure, mean heart rates (HR) and stroke volume indices (SVI) changes in patients' baseline condition (Control), after angiotensin infusion (AGT) and after sublingual administration of nitroglycerin (NTG). $S P$, systolic pressure; DP, diastolic pressure; EDP, left ventricular enddiastolic pressure. Values are given as mean $\pm S D$. ${ }^{*} p<0.01$.

Table 2 Changes in the AI, TPmax, TPo and Tau

\begin{tabular}{llllc}
\hline \hline & \multicolumn{1}{c}{ AI } & $\begin{array}{c}\text { Tpmax } \\
(\mathrm{msec})\end{array}$ & $\begin{array}{c}\text { Tpo } \\
(\mathrm{msec})\end{array}$ & $\begin{array}{c}\text { Tau } \\
(\mathrm{msec})\end{array}$ \\
\hline Control & $1.61 \pm 1.14$ & $279 \pm 31$ & $371 \pm 26$ & $49 \pm 4$ \\
AGT & $2.08 \pm 1.11^{*}$ & $293 \pm 28^{*}$ & $391 \pm 26^{*}$ & $54 \pm 4^{*}$ \\
NTG & $1.27 \pm 1.14^{*}$ & $259 \pm 25^{*}$ & $354 \pm 27^{*}$ & $45 \pm 5^{*}$ \\
\hline
\end{tabular}

The mean values of the augmentation index (AI), the times from onset of the $R$ wave in electrocardiogram to peak $L V$ pressure (TPmax) and to minimal dP/dt (TPo), and the time constants of isovolumetric LV pressure decay (Tau) in patients' baseline condition (Control), after angiotensin infusion (AGT) and after sublingual administration of nitroglycerin $(N T G)$.Values are given as mean $\pm S D .{ }^{*} p<0.01$.

obtained from the pressure data.

The Time of Peak LV Pressure (TPmax): The time from the onset of the $\mathrm{R}$ wave on electrocardiogram to peak LV pressure (TPmax) was obtained from the pressure data.

AI: The AI is defined as the ratio of the height of the late systolic shoulder/peak to that of the early systolic shoulder/peak in the pulse (Fig 2)! ${ }^{14}$

\section{Statistical Analysis}

All data were expressed as mean $\pm 1 \mathrm{SD}$. Comparisons with the control state were made using ANOVA with Fisher's protected least significant difference analysis. A p value less than 0.05 was considered significant difference. Correlation coefficients were computed based on leastsquares linear regression analysis.

\section{Results}

\section{Hemodynamics}

The mean hemodynamic data for the baseline, angiotensin, and nitroglycerin conditions are shown in Table 1. The mean heart rates during the three loading conditions were similar because of right atrial pacing. In contrast, both the mean ascending aortic systolic pressure and ascending aortic diastolic pressure increased by $25 \%$ and $22 \%$ during the infusion of angiotensin $(\mathrm{p}<0.01$ for both) and decreased by $14 \%$ and $4 \%$ during the sublingual administration of nitroglycerin ( $\mathrm{p}<0.01$ for both) compared with baseline condition. The mean LV end diastolic pressure increased by $53 \%$ during the infusion of angiotensin $(\mathrm{p}<0.01)$ and decreased by $33 \%$ during the sublingual administration of nitroglycerin $(\mathrm{p}<0.01)$ compared with baseline condition. The mean stroke volume index values were not significantly affected by these permutations in loading conditions.

Changes in the AI, TPmax, TPo and Tau

The mean values of the AI, TPmax, TPo and Tau for the 


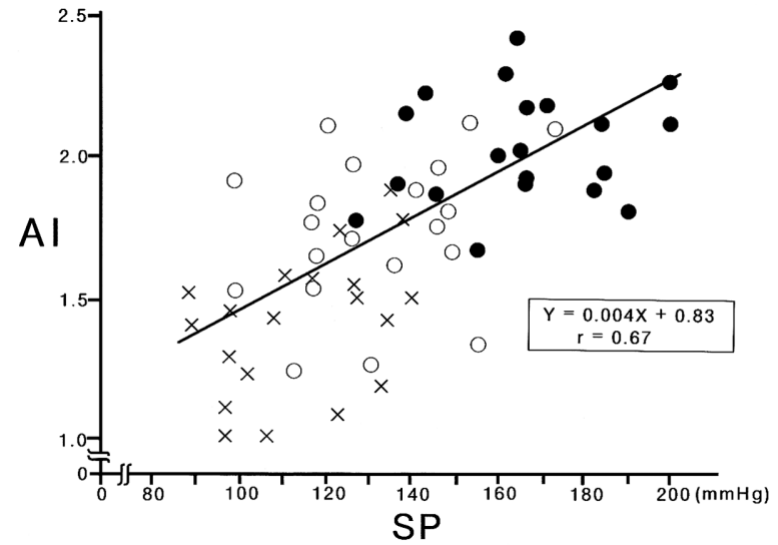

Fig 3. Correlation between systolic pressure (SP) and the augmentation index (AI) under baseline conditions $(\mathrm{O})$, after angiotensin infusion (๑) and after sublingual administration of nitroglycerin $(x)$. The AI increased proportionally with ascending aortic systolic pressure $(\mathrm{p}<0.001, \mathrm{r}=0.67)$.

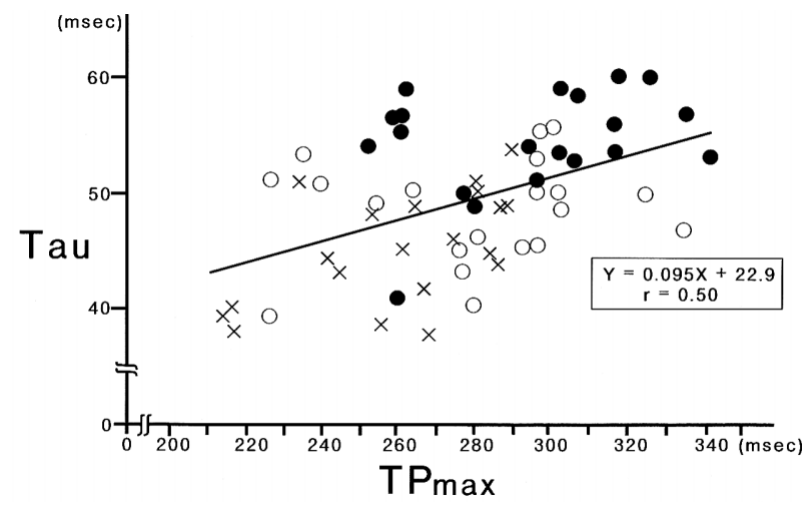

Fig 4. Correlation between the times from onset of the $\mathrm{R}$ wave on electrocardiogram to peak LV pressure (TPmax) and the time constants of isovolumic LV pressure decay (Tau) under baseline conditions $(\bigcirc)$, after angiotensin infusion $(\bullet)$ and after sublingual administration of nitroglycerin $(x)$. Tau prolonged proportionally with delay of TPmax $(\mathrm{p}<0.001, \mathrm{r}=0.50)$.

baseline, angiotensin, and nitroglycerin conditions are shown in Table 2 . The mean values of the AI increased during infusion of angiotensin $(p<0.01)$ and decreased during the sublingual administration of nitroglycerin $(\mathrm{p}<0.01)$ compared with the baseline condition. Both the mean TPmax and TPo were delayed during infusion of angiotensin $(p<0.01$ for both) and accelerated during the sublingual administration of nitroglycerin $(p<0.01$ for both) compared with the baseline condition. Tau was prolonged during infusion of angiotensin $(p<0.01)$ and shortened during the sublingual administration of nitroglycerin $(\mathrm{p}<0.01)$ compared with the baseline condition. The AI increased proportionally with ascending aortic systolic pressure $(\mathrm{p}<0.001, \mathrm{r}=0.67)$ (Fig 3). However, neither TPmax nor TPo correlated with ascending aortic systolic pressure. Tau was prolonged proportionally with the delay in TPmax $(\mathrm{p}<0.001, \mathrm{r}=0.50)$, TPo $(\mathrm{p}<0.001$, $\mathrm{r}=0.57)$, and increasing ascending aortic AI $(\mathrm{p}<0.001$, $\mathrm{r}=0.64$ ) (Figs 4,5,6). Tau, however, did not directly correlate with ascending aortic systolic pressure.

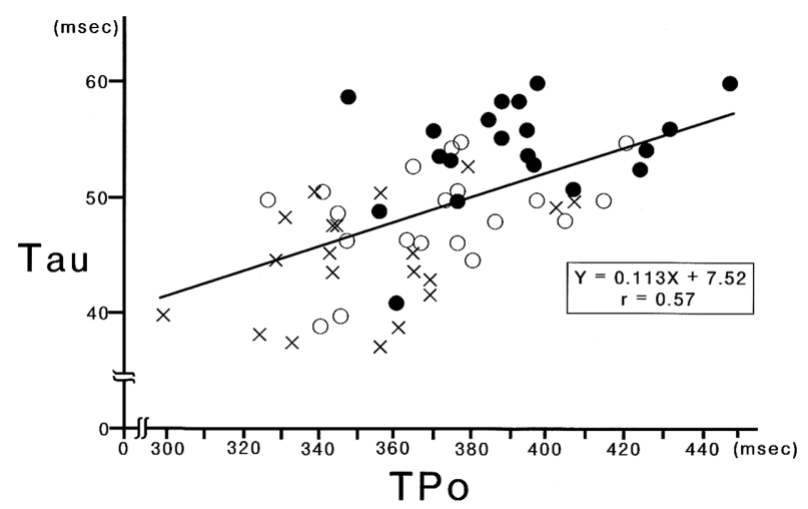

Fig 5. Correlation between the times from onset of the $\mathrm{R}$ wave on electrocardiogram to minimal $\mathrm{dP} / \mathrm{dt}$ (TPo) and the time constants of isovolumic LV pressure decay (Tau) under baseline conditions $(O)$, after angiotensin infusion ( $(\mathbf{)}$ and after sublingual administration of nitroglycerin $(x)$. Tau prolonged proportionally with delay of TPo $(\mathrm{p}<0.001, \mathrm{r}=0.57)$.

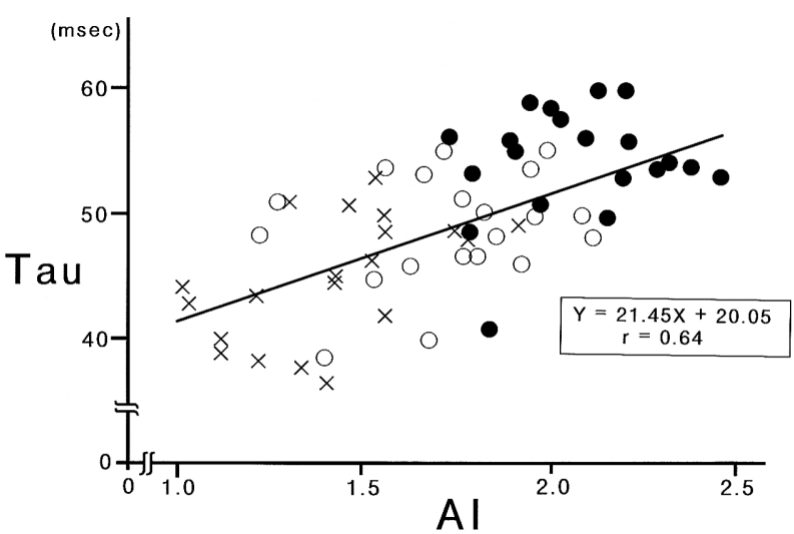

Fig 6. Correlation between the augmentation index (AI) and the time constants of isovolumic LV pressure decay (Tau) under baseline conditions $(O)$, after angiotensin infusion $(\mathbf{\bullet})$ and after sublingual administration of nitroglycerin $(x)$. Tau prolonged proportionally with increasing ascending aortic $\mathrm{AI}(\mathrm{p}<0.001, \mathrm{r}=0.64)$.

\section{Discussion}

\section{Heart Rate and LV Relaxation}

An increased HR has been reported to accelerate the rate of isovolumic LV pressure decline, not only in animal experiments $^{4,8}$ but also in clinical investigations?, 10 In the present study, the HR was kept constant by right atrial pacing, which made it possible to perform the experiment without any influence of the HR on Tau.

\section{Systolic Pressure and LV Relaxation}

Several investigators reported that Tau is prolonged by the increased blood pressure during methoxamine treatment $^{2}$ and by the abrupt occlusion of the descending thoracic aorta! However, other investigators reported that Tau is accelerated by the increased blood pressure caused by norepinephrine 4 and dobutamine treatment? This inverse relation may be based on changes in the loading pattern or the contour of the ascending aortic systolic pressure wave. In the ascending aortic systolic pressure wave, an inflection point (anacrotic notch) corresponds to the peak of ejection flow velocity from LV 13 The pressure wave up to this point 
is categorized as an early systolic component, which is mainly caused by LV ejection, and the pressure wave after this point is a late systolic component, which is mainly caused by the peripheral reflection wave (Fig 2). Methoxamine treatment and the abrupt occlusion of the descending thoracic aorta increase the reflection wave, which leads to an increase in the late systolic component of ascending aortic systolic pressure, whereas norepinephrine and dobutamine treatment increase LV ejection, which leads to an increase in early systolic component of ascending aortic systolic pressure.

\section{Loading Patterns and LV Relaxation}

Hori et al clamped the ascending or descending aorta of anesthetized thoracotomized dogs, and observed changes in LV relaxation by increasing the early peak and late peak systolic pressure? They reported that the increase in early peak systolic pressure resulted in the prolonged onset of the LV isovolumic relaxation period and a slightly prolonged Tau, whereas an increase in late peak systolic pressure resulted in earlier onset of the $\mathrm{LV}$ isovolumic relaxation period and a remarkably prolonged Tau. They indicated that the loading sequence was an important factor that affects LV relaxation, because two different loads caused different levels of change in Tau.

Therefore, it has been confirmed that an increase in late peak systolic pressure results in the earlier onset of the LV isovolumic period and a prolonged Tau. In our present clinical study, 18 an increase in late peak systolic pressure, described as an increase in the AI, caused a delay in onset and a prolongation of Tau. These results, showing that Tau was prolonged by an increase in late peak systolic pressure, were similar to those in the animal experiment of Hori et al? However, in contrast to that study, the onset of the LV isovolumic period due to increased late peak systolic pressure was prolonged. It has already been reported by Lewis et al that the influence of afterload on ejection time differs between clinical observation and animal experiments $!^{19}$ If the ventricular properties are constant, the timing of end ejection should be accelerated by an increase of systolic pressure, as in the results obtained in animal experiments. The timing of end ejection, however, is prolonged in the clinical setting. This may be because some kind of compensation mechanism, such as hypertrophy caused by chronic load, is generated in human cardiac muscle, resulting in prolongation of the ejection time.

\section{Relation Between AI and LV Relaxation}

The AI in pulse pressure represents the percentage of the late systolic component to the early systolic component, or the percentage of peripheral reflection wave to ejection pressure wave (Fig 2). In the present study, the AI increased with the elevated pressure due to peripheral vasoconstriction caused by angiotensin, and it decreased with the decrease in blood pressure due to peripheral vasodilation caused by nitroglycerin. There was no relationship between systolic pressure and Tau, but a relationship was recognized between the AI and Tau. This indicates that clinically LV relaxation is possibly influenced not merely by changes in total systolic pressure but also by a pressure change mainly in the late systolic component. The AI value increases with acceleration of the reflection wave, which is caused by organic arterial wall stiffness (mainly due to arterial sclerosis) and mechanical tension of the vascular wall, (mainly due to intravascular pressure). It generally increases with age.11,12 Some reports state that LV relaxation indices, such as LV diastolic flow velocity waveforms, become disturbed with aging. ${ }^{20,21}$ In such cases, LV relaxation may be chronically affected by changes in afterload mainly composed of reflective waves.

\section{Defining Factors in LV Relaxation Properties}

Our present study suggested that LV relaxation is influenced by prolonged TPmax and TPo caused by afterload changes, mainly consisting of reflection waves, and by changes in blood pressure increase patterns such as increases in the AI. Hori et al reported in other experiments with canine cardiac muscle that the time of end ejection (TPo) is related to the properties of LV relaxation, and that the more TPo is prolonged, the more Tau is shortened? However, our results concerning the TPo-Tau relationship contradict those of Hori et al: ${ }^{7}$ the more TPo is prolonged, the more Tau is prolonged. Therefore, the time of end ejection is, clinically, not likely to be the main factor that defines the properties of LV relaxation. On the other hand, our results indicating that a prolonged Tau is related to the delay in the timing of maximum late systolic pressure were consistent with those of Hori et al?

Increased pressure, mainly composed of the late systolic component, is associated with prolonged TPmax and increased AI, and this prolongs Tau. How can this result be explained? According to the concept of shortening deactivation,2 an increase in the end-systolic volume results from increased late systolic pressure; that is, prolonged shortening of the cardiac muscle may cause the prolonged relaxation rate. On the other hand, the shortening of the cardiac muscle itself has an influence over calcium sensitivity for troponin C ${ }^{23}$ Late systolic load on the cardiac muscle may affect the cardiac muscle shortening process, which leads to a change in calcium kinetics that prolongs the relaxation rate. Further investigations are needed to confirm this.

\section{Limitations of the Study}

The present study included patients with ischemic heart disease and myocarditis. In such cases, the time constants of isovolumic relaxation may be affected by ischemia ${ }^{4,24}$ and the nonuniformity of relaxation ${ }^{25}$ produced by altered loading conditions. However, 3 cases of chest pain syndrome, considered to have almost normal left ventricles, showed load dependence of the rate of isovolumic LV relaxation. In the present study, both nitroglycerin and angiotensin had effects on LV end-diastolic pressure as well as LV systolic pressure. These agents, therefore, may not be pure methods for altering late systolic pressure changes.

\section{Conclusions}

An increase in systolic blood pressure caused by vasoconstriction prolonged the onset and the rate of isovolumic LV pressure decline, whereas a decrease in the systolic blood pressure caused by vasodilation shortened the onset and the rate of isovolumic LV pressure decline. We conclude that late systolic pressure augmentation in the ascending aorta is one important factor that influences the rate of isovolumic LV pressure decline in humans. Aging causes sclerosis of the arterial wall and increases the reflection wave, which leads to an increase in the late systolic component of systolic pressure. In such cases, LV relaxation may be chronically affected by changes in afterload, mainly composed of reflective waves. 


\section{Acknowledgments}

We are grateful to the staff and technicians of the catheterization laboratories of the Tokyo Medical College Hospital. The authors are indebted to Professor J. Patrick Barron of the Tokyo Medical College Hospital for his review of the manuscript.

\section{References}

1. Blaustein AS, Gaasch WH: Myocardial relaxation. VI. Effects of beta-adrenergic tone and asynchrony on LV relaxation rate. Am J Physiol 1983; 244: 417-422

2. Gaasch WH, Blaustein AS, Andrias CW, Donahue RP, Avitall B: Myocardial relaxation.II. Hemodynamic of rate of left ventricular isovolumic pressure decline. Am J Physiol 1980; 239: 1-6

3. Hori M, Inoue M, Kitakaze M, Tsujioka K, Ishida Y, Fukunami M, et al: Loading sequence is a major determinant of afterload-dependent relaxation in intact canine heart. Am J Physiol 1985; 249: 747 754

4. Weiss JL, Frederiksen JW, Weisfeldt ML: Hemodynamic determinants of the time-course of fall in canine left ventricular pressure. $J$ Clin Invest 1976; 58: $751-760$

5. Starling MR, Montgomery BS: Load independence of the rate of isovolumic relaxation in man. Circulation 1987; 76: 1274-1281

6. Zile MR, Nishimura RA, Gaasch WH: Hemodynamic loads and left ventricular diastolic function: Factors affecting the indices of isovolumetric and auxotonic relaxation. In: Gaasch WH, LeWinter MM editors. Left ventricular diastolic dysfunction and heart failure. Philadelphia: Lea \& Febiger, 1994: 219-242

7. Hori M, Kitakaze M, Ishida Y, Fukunami M, Kitabatake A, Inoue M, et al: Delayed end ejection increases isovolumic ventricular relaxation rate in isolated perfused canine hearts. Circ Res 1991; 68: 300308

8. Frederiksen JW, Weiss JL, Weisfeldt ML: Time constant of isovolumic pressure fall: determinants in the working left ventricle. Am J Physiol 1978; 235: 701-706

9. Fioretti P, Brower RW, Meester GT, Serruys PW: Interaction of left ventricular relaxation and filling during diastole in human subjects. Am J Cardiol 1980; 46: 197-203

10. Rosseau MF, Pouleur H, Detry J-MR, Brasseur LA: Relationship between changes in left ventricular inotropic state and relaxation in normal subjects and in patients with coronary artery disease. Circulation 1981; 64: 736-743
11. Murgo JP, Westerhof N, Giolma JP, Altobelli SA: Aortic input impedance in normal man: relationship to pressure wave shapes. Circulation 1980; 62: 105-116

12. Takazawa K: A clinical study of the second component of left ventricular systoric pressure. J Tokyo Med Coll 1987; 45: 256-270

13. Kelly RP, Hayward CS, Avolio AP, O'Rourke MF: Non-invasive determination of age-related changes in the human arterial pulse. Circulation 1989; 62: 105-116

14. Takazawa K, Tanaka N, Takeda K, Kurosu F, Ibukiyama C: Underestimation of vasodilator effects of nitroglycerin by upper limb blood pressure. Hypertension 1995; 26: 520 - 523

15. Yaginuma T, Noda T, Tsuchiya M, Takazawa K,Tanaka H, Kotoda $\mathrm{K}$, et al: Interaction of left ventricular contraction and aortic input impedance in experimental and clinical studies. Jpn Circ J 1985; 49: 206-214

16. Abel FL: Maximal negative $\mathrm{dP} / \mathrm{dt}$ as an indicator of end systole. Am J Physiol 1981; 240: 676-679

17. Martin G, Gimeno JV, Ramirez A, Cosin J, Baguena J: Effects of high-frequency harmonics on cardiac relaxation indices. Am J Physiol 1981; 240: 669-680

18. Yabe K, Takazawa K, Maeda K, Fujita M, Sakai T, Chiharu Ibukiyama: The influence of ascending aortic augmentation index (reflection coefficient) to left ventricular relaxation (abstract). Circulation 1991; 84: 565

19. Lewis RP, Leighton RF, Forester WF,Weissler AM: Systolic time intervals. In: Weissler AM editor. Noninvasive cardiology. New York: Grune \& Stratton, 1974: 301-368

20. Spirito $P$, Maron B: Influence of aging on doppler echocardiographic indices of left ventricular function. Br Heart J 1988; 59: 672-679

21. Bryg RJ, Williams GA, Labovitz AJ: Effect of aging on left ventricular diastolic filling in normal subjects. Am J Cardiol 1987; 59: 971 974

22. Jewell BR, Wilkie DR: The mechanical properties of relaxing muscle. J Physiol (London) 1960; 152: 30-47

23. Allen, DG, Kurihara S: Length changes during contraction affect the intracellular $[\mathrm{Ca} 2+]$ of heart muscle. J Physiol (London) 1981; 310: $75-76$

24. Palacios I, Newell JB, Powell WJ: Effect of acute global ischemia on diastolic relaxation in canine hearts. Am J Physiol (Heart Circ Physiol) 1978; 235: 720-727

25. Stefan S, Volker BF, Volker T: Afterload dependent prolongation of left ventricular relaxation: impotance of asynchrony. Cardiovasc Res 1992; 26: 631-637 